\title{
SHEAR-WAVE VELOCITY AT THE BASE OF THE MANTLE FROM PROFILES OF DIFFRACTED $S H$ WAVES
}

\author{
By Emile A. OKal* and Robert J. Geller $\dagger$
}

\begin{abstract}
Profiles of $\mathrm{SH}$ waves diffracted around the core $(\mathrm{Sd})$ for three deep events at stations across North America and the Atlantic $\left(\Delta=92^{\circ}\right.$ to $\left.152^{\circ}\right)$ are used to determine the properties of the lower mantle in the vicinity of the core-mantle boundary (CMB). The $S$-wave velocity above the CMB is found to be $\beta_{c}=7.22$ $\pm 0.1 \mathrm{~km} / \mathrm{sec}$, in agreement with gross earth models, but higher than previously reported values from direct measurements of Sd. The frequency imdependence of the $S d$ ray parameter argues strongly against the possibility of a low-velocity zone immediately above the core mantle boundary.

We compute synthetic seismograms for $S d$ by summing normal modes. A comparison of the present data with a synthetic profile for earth model 1066A gives excellent agreement at periods greater than $\mathbf{4 5}$ seconds. Synthetics for other models are used to substantially constrain the possibility of significant rigidity of the uppermost layer of the core.
\end{abstract}

\section{INTRODUCTION}

The seismic properties of the deepest parts of the mantle, in the vicinity of the core-mantle boundary (CMB) have been the subject of extensive, and at times controversial, studies. Individual observations of a decrease in $S$-wave velocity near the CMB (Cleary, 1969; Bolt et al., 1970) and of an associated low-Q zone (Mikumo and Kurita, 1968) have been reported. Some data obtained from free oscillation $Q$ studies (Anderson and Hart, 1978) have supported these reports, although gross earth models (Gilbert and Dziewonski, 1975; Anderson and Hart, 1976) have usually failed to yield a low shear velocity zone at the base of the mantle. Also, an increase in $S$-wave velocity above the CMB has been proposed by Mitchell and Helmberger (1973) on the basis of $S c s$ to $S$ amplitude ratios.

The seismic properties just above the CMB have significant geophysical implications. A low- $Q$, low- $S$ velocity zone might be related to efficient heat transfer across the boundary, and possibly, also, to a difference in chemical content in the deepest shells of the mantle. In turn, the composition of the deepest mantle bears directly on our understanding of the differentiation process in the Earth and other planets (Jacobs, 1975).

Most of the seismic evidence for a low-S wave velocity zone at the base of the mantle comes from the study of $S H$ waves diffracted along the CMB into the shadow zone for direct $S$ waves. We will call this phase $S d$. Cleary et al. (1967), Cleary (1969), Bolt et al. (1970), and Hales and Roberts (1970) reported values obtained from $S d$ studies of $7.06,6.8,6.99$, and $6.78 \mathrm{~km} / \mathrm{sec}$, respectively, for the $S$-wave velocity at the base of the mantle, $\beta_{c}$, as opposed to Jeffreys' 7.30 , Anderson and Hart's 7.23, or Gilbert and Dziewonski's 7.25. Mondt (1977), using the techniques of Scholte (1956), Richards (1970), and Chapman and Phinney (1972), suggested a value of $\beta_{c}=7.03 \mathrm{~km} / \mathrm{sec}$ from the amplitude decay of $S d$ waves with distance.

The primary purpose of this paper is to resolve the question of the $S$ velocity at the base of the mantle, by using a set of three profiles of high-quality $S d$ data,

\footnotetext{
* Present address: Department of Geology and Geophysics, Yale University, New Haven, Connecticut 06520.

$\dagger$ Present address: Department of Geophysics, Stanford University, Stanford, California 94305.
} 
sampling the CMB in two different geographical areas. By measuring $d T / d \Delta$ of $S d$ using two different methods, we obtain an $S$-wave velocity $\beta_{c}=7.22 \pm 0.1 \mathrm{~km} / \mathrm{sec}$ immediately above the CMB. As this result rests on geometrical optics, we conduct some synthetic seismogram experiments at long periods, using normal mode summation, to confirm this result.

The synthetic seismograms for Gilbert and Dziewonski's (1975) model 1066A agree very well with the observed profiles. In contrast, observed amplitudes for $S d$ at large distances $\left(\Delta>120^{\circ}\right)$ are inconsistent with models including a small but finite $(\beta=0.73-2 \mathrm{~km} / \mathrm{sec})$ rigidity in the core, in a layer extending $25 \mathrm{~km}$ or more below the CMB.

The existence of $S d$ was mentioned by Gutenberg and Richter (1935), but the first reported data are found in Lehmann (1953). Cleary et al. (1967) first measured the apparent slowness of the phase. Their value $(p=8.61 \mathrm{sec} / \mathrm{deg})$ corresponds to $\beta_{c}=7.06 \mathrm{~km} / \mathrm{sec}$ for a radius of the core, $r_{c}=3485 \mathrm{~km}$, through the relation

$$
\beta_{c}=(\pi / 180) \cdot r_{c} / p
$$

Later, Cleary (1969) proposed $\beta_{c}=6.8 \mathrm{~km} / \mathrm{sec}$. However, both of these studies used a single station (Canberra, Australia) and several earthquakes at various azimuths. Errors in the earthquakes' hypocentral parameters can cause large uncertainties in

TABLE 1

Seismic Events Used in This Study

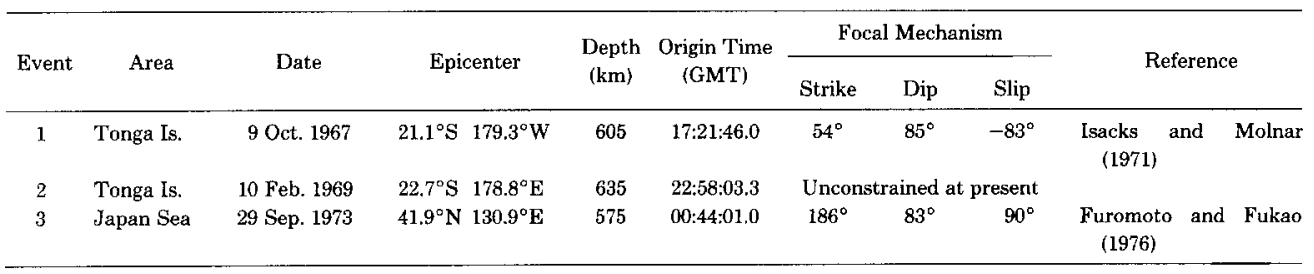

the final results. The opposite experiment (using a single event recorded along a profile of stations at the same azimuth) has the advantage of eliminating epicentral errors, and of providing a fairly uniform wave shape. Bolt et al. (1970) used such a profile of diffracted $S H$ waves for the (shallow) August 31, 1968 Dasht-e-Bayāz (Iran) earthquake, and obtained $\beta_{c}=6.99 \pm 0.1 \mathrm{~km} / \mathrm{sec}$. However, $S d$ picks for a shallow event can be complicated by shallow structure. Also, the focal mechanism of the 1968 event [strike-slip on a nearly vertical fault; Niazi (1969)] was quite unfavorable to excitation of $S d$, especially at the particular azimuths used by Bolt et al. In this study, we use high-quality $S d$ profiles for three deep earthquakes (Tonga, 1967 and 1969; Japan Sea, 1973). Using deep earthquakes eliminates possible problems caused by later phases. Also, the focal mechanisms of the 1967 Tonga and 1973 Japan Sea events (for which accurate focal mechanisms have been published) excite $S d$ efficiently. The mechanism is also apparently favorable for the 1969 Tonga event.

\section{$S d$ Data Set}

Table 1 gives the source parameters of the three events we used for the $S d$ profiles. The focal mechanism of event 1 (October 9, 1967) is given by Isacks and Molnar (1971), and that of event 3 (September 29, 1973), by Furumoto and Fukao 
(1976). No focal mechanism has been published for event 2, and the $P$-wave first motions are insufficient to properly constrain it. Table 2 lists the stations we used with their distances, azimuths, and back azimuths.

The theoretical expression for the amplitude of a ray leaving the focal sphere is given by Chung and Kanamori (1976)

$$
R^{S H}=-q_{L} \cos i_{h}-p_{L} \sin i_{h}
$$

where $q_{L}$ and $p_{L}$ are the radiation-pattern coefficients defined by Kanamori and Cipar (1974), and depend on fault dip and slip angles, and on the azimuth of the station from the fault strike; $i_{h}$ is the takeoff angle at the focal sphere. Although $R^{S H}$ at grazing incidence at the CMB cannot be used to obtain the absolute

TABLE 2

Seismic Records Used in This Study

\begin{tabular}{|c|c|c|c|c|c|}
\hline Code & Station & Instrument & Distance $\left(^{\circ}\right)$ & Azimuth $\left(^{\circ}\right)$ & $\begin{array}{c}\text { Back Azimuth } \\
\left({ }^{\circ}\right)\end{array}$ \\
\hline \multicolumn{6}{|c|}{ Event 1} \\
\hline LUB & Lubbock, Texas & WWSSN & 91.64 & 54.7 & 245.9 \\
\hline FLO & Florissant, Mo. & WWSSN & 102.18 & 53.2 & 252.9 \\
\hline SCP & State College, Penna. & WWSSN & 112.00 & 53.5 & 260.9 \\
\hline PAL & Palisades, N.Y. & Press-Ewing & 115.00 & 53.7 & 263.4 \\
\hline WES & Weston, Mass. & WWSSN & 117.06 & 52.4 & 265.7 \\
\hline HAL & Halifax, N.S. & Canadian & 122.72 & 50.0 & 272.3 \\
\hline STJ & St. John's, Nfld. & Canadian & 129.83 & 45.3 & 282.9 \\
\hline PDA & Ponta Delgada, Azores & WWSSN & 151.86 & 48.6 & 299.3 \\
\hline \multicolumn{6}{|c|}{ Event 2} \\
\hline LUB & Lubbock, Texas & WWSSN & 94.06 & 55.5 & 245.6 \\
\hline OXF & Oxford, Miss. & WWSSN & 104.04 & 58.4 & 252.2 \\
\hline AAM & Ann Arbor, Mich. & WWSSN & 110.44 & 51.8 & 257.7 \\
\hline SCP & State College, Penna. & WWSSN & 114.42 & 54.3 & 260.6 \\
\hline WES & Weston, Mass. & WWSSN & 119.49 & 53.3 & 265.5 \\
\hline STJ & St. John's, Nfld. & Canadian & 132.27 & 45.9 & 283.1 \\
\hline \multicolumn{6}{|c|}{ Event 3} \\
\hline $\mathrm{BEC}$ & Hamilton, Bermuda & WWSSN & 104.56 & 13.5 & 348.1 \\
\hline SJG & San Juan, P.R. & WWSSN & 118.12 & 18.4 & 345.7 \\
\hline TRN & Trinidad, Trinidad \& Tobago & WWSSN & 126.37 & 15.0 & 348.6 \\
\hline
\end{tabular}

amplitude of $S d$, it provides a useful estimate of the relative amplitude of $S d$ for different events and profile azimuths. The optimal fault geometry for $S d$ profiles $\left(R^{S H}=1\right)$ excited by a deep earthquake $\left(i_{h}=30^{\circ}\right)$ is a vertical fault with a slip angle of about $60^{\circ}$ and stations at the azimuth of the fault strike. Such a mechanism causes a large amount of shear energy to be radiated downward. Large amplitudes of downgoing $S$ waves also lead to excellent multiple $S c S$ phases. The three earthquakes we used were previously used in multiple $S c S$ studies (Okal and Anderson, 1975; Yoshida and Tsujiura, 1975; Sipkin and Jordan, 1976). Also, the 1967 Tonga event was used in a recent SKS study (Kind and Muller, 1977). The radiation pattern factors for the two events whose mechanisms are known are $R^{S H}$ $=0.901$ (event 1 ) and $R^{S H}=0.811$ (event 3 ) at the azimuths of our profiles. Thus, the quality of the data is considerably better than in previous studies. (For the Dasht-e-Bayāz event recorded at American stations, $R^{S H}=0.08$.) 


\section{Data Analysis}

Records were rotated into $S H$ polarization whenever the station's back azimuth was more than $10^{\circ}$ away from natural polarization. Records at Canadian stations and PAL were equalized to the amplitude and phase response of the WWSSN instrument. We will concentrate on event 1 , because of the excellent quality of the data, and the well-determined focal mechanism; however, all of our results are confirmed by events 2 and 3 .
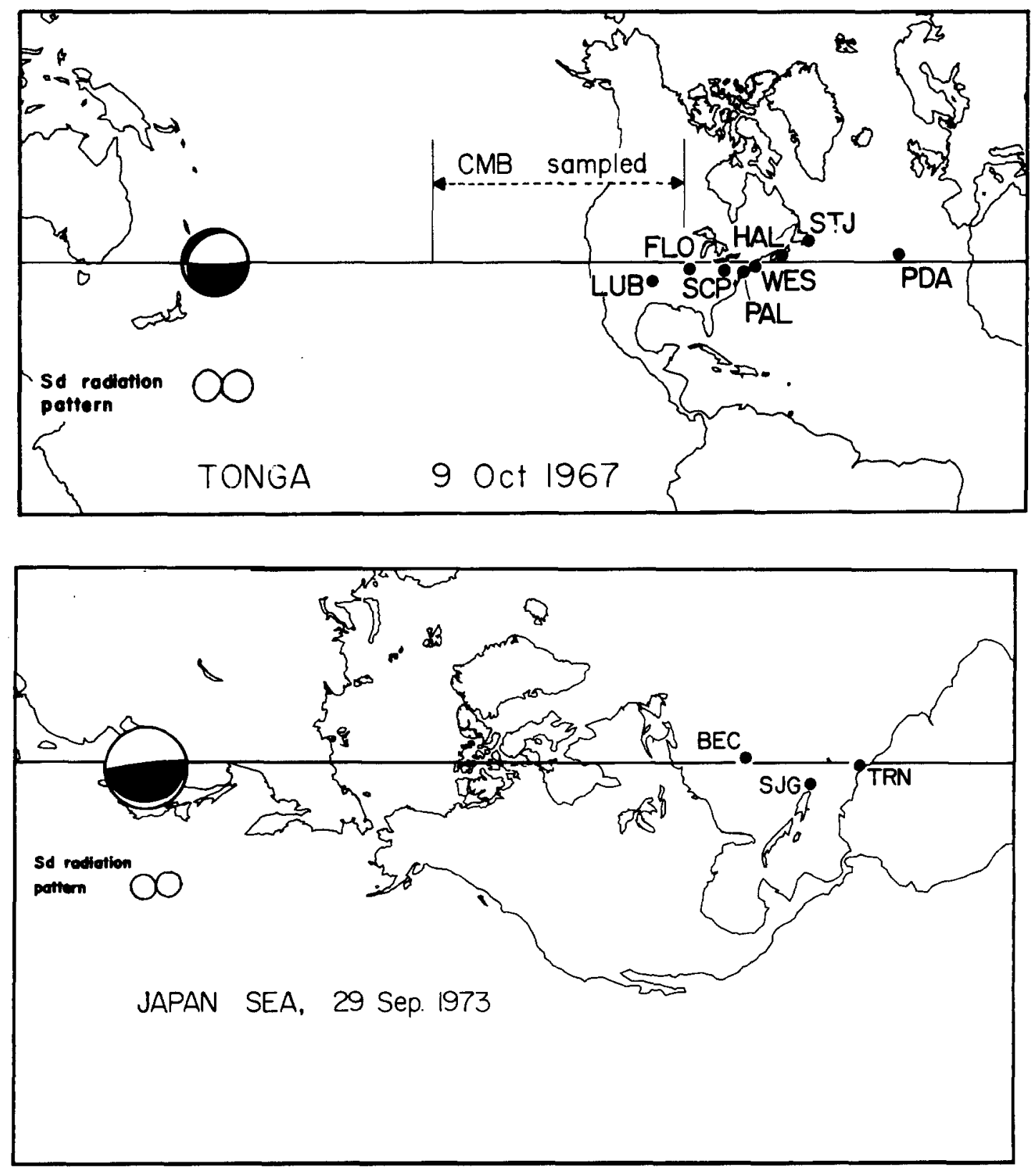

Fig. 1. Top: Map of the profile for event 1. This map is a Mercator projection whose base is the mean great circle linking the epicenters and the stations. Also shown are the earthquake focal mechanism, the theoretical radiation pattern for $S d$, and the portion of CMB sampled by the profile. Bottom: Same as top for event 3. 

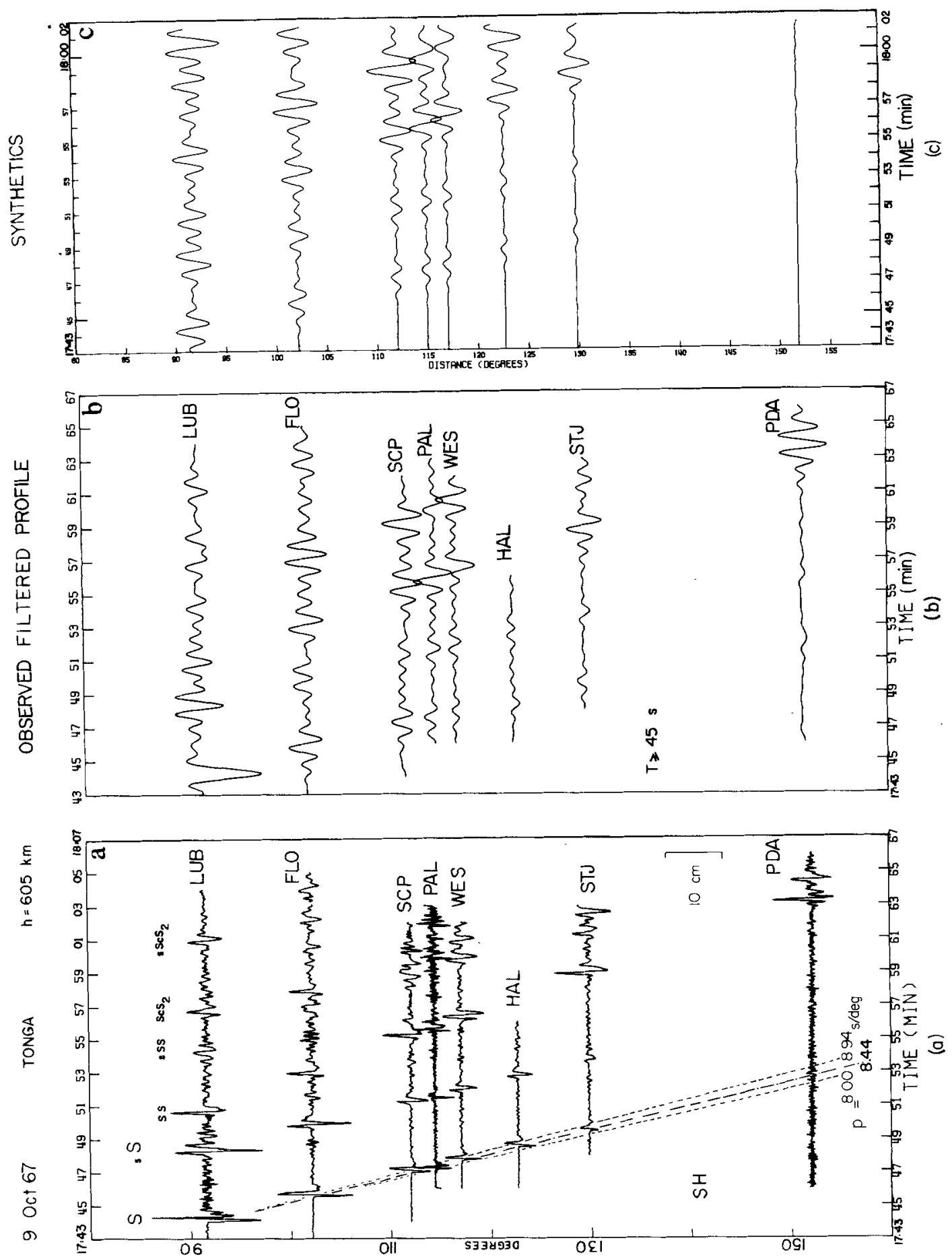

FIG. 2. (a) Observed profile for event 1 . The seismograms have been rotated into $S H$ polarization, and equalized to a standard magnification of 1500 (and to the WWSSN instrument for Canadian stations and PAL). The oblique lines show the result of alignment of the onsets with a straight edge $\left(p_{0}=8.44 \mathrm{sec} /\right.$ $\mathrm{deg}$ ) and lines of constant slowness corresponding to 6.8 and $7.6 \mathrm{~km} / \mathrm{sec}$ at the CMB. Note that a lowvelocity zone model predicts residuals of about $12 \mathrm{sec}$ at STJ, much larger than the observed values ( $\cong 2$ sec), which correspond to station corrections due to crust and upper mantle structure. (b) Observed profile filtered at $T \geqq 45$ sec. Note that $S d$ is detected all the way to PDA. (c) Synthetics obtained by adding up all modes with $T \geqq 45 \mathrm{sec}$. 
Figure 1 shows the profile for events 1 and 3. The data for event 1 are shown on Figure 2a. $S d$ has a very sharp onset at all stations up to STJ. The identification of $S d$ at PDA is more difficult on the unfiltered record, because of the smaller signal and larger noise level of this island station, but $S d$ is clear on the filtered record (Figure $2 \mathrm{~b}$ ). The phase $s S d$ is also present on all records.

Given the sharpness of the onset, a visual alignment of the first arrivals can be made using only a straight edge. The result, shown on Figure $2 \mathrm{a}$, is $p_{0}=8.44 \mathrm{sec} /$
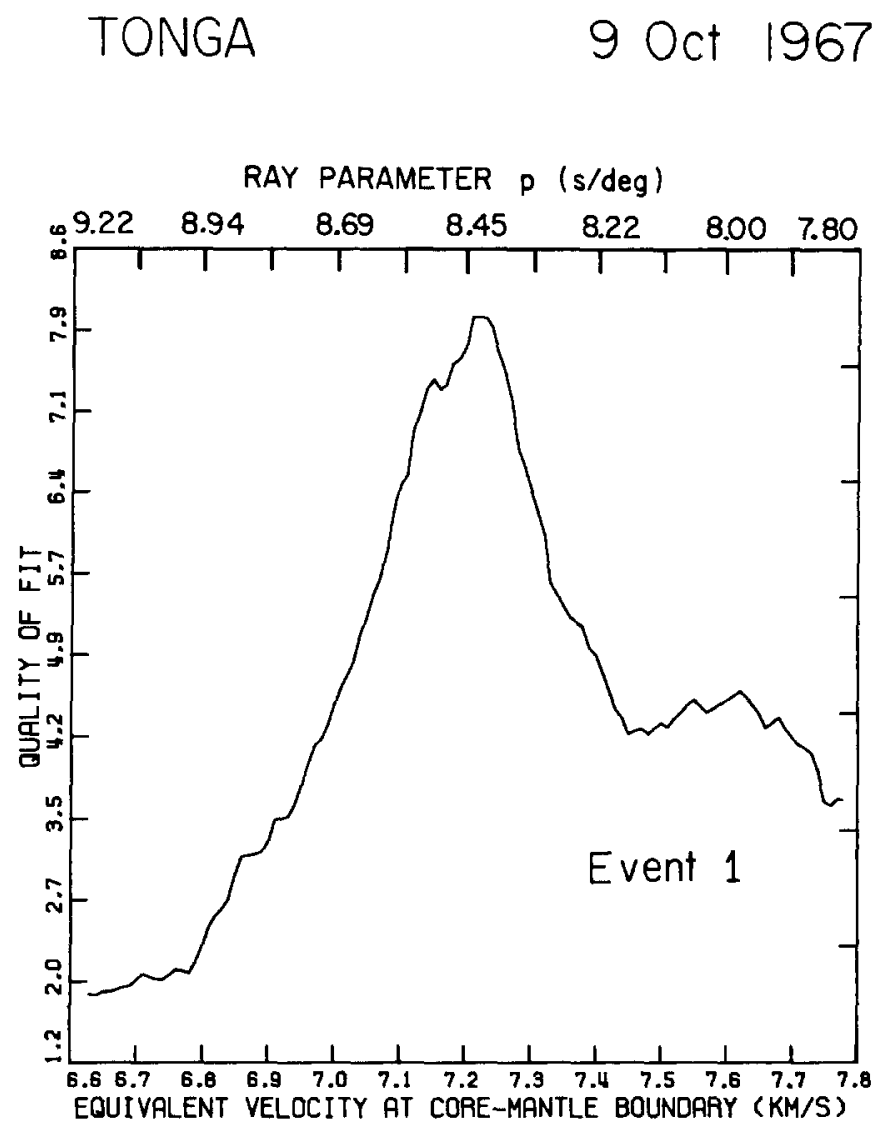

FIG. 3. Variation of the cross-correlation $F$ as a function of slowness $p$ (or equivalently shear-wave velocity at the CMB), for event 1 .

deg, or $\beta_{c}=7.21 \mathrm{~km} / \mathrm{sec}$. Estimating the error bar for this number is difficult, although values of $8.00\left(\beta_{c}=7.6\right)$ and $8.94\left(\beta_{c}=6.8\right)$ (shown on Figure 1) clearly do not fit the data. The residuals in reduced travel time $\left(T-p_{0} \Delta\right)$ are on the order of $\pm 2 \mathrm{sec}$, whereas models with a sharp low-velocity zone, such as the one proposed by Cleary (1969) would lead to residuals on the order of $12 \mathrm{sec}$ at $130^{\circ}$, inconsistent with the data on Figure 2a. Residuals on the order of $2 \mathrm{sec}$ are compatible with $S$ wave station corrections, as studied, for example, by Hales and Roberts (1970).

A second measurement of $\beta_{\mathfrak{c}}$ was made by cross correlation of the various records. After the $S d$ phases were isolated and the signal energy normalized to unity, we computed the cross-correlation functions $y_{i} \oplus y_{j}$ between the signals. If $\Delta_{i}$ is the epicentral distance of the $i$ th station, we then compute 


$$
F=\sum_{i} \sum_{J} y_{i}\left(t-p \cdot \Delta_{i}\right) \oplus y_{j}\left(t-p \cdot \Delta_{j}\right)
$$

for various values of the slowness $p$. It is easily seen that

$$
F=\sum_{i} \sum_{j}\left(y_{i} \oplus y_{j}\right)\left(t_{i j}\right)
$$

where the cross correlation is taken for the lag: $t_{i j}=p \cdot\left(\Delta_{i}-\Delta_{j}\right) . F$ is a measure of the quality of the fit between all 8 traces when time-lagged with a slowness $p$. Figure
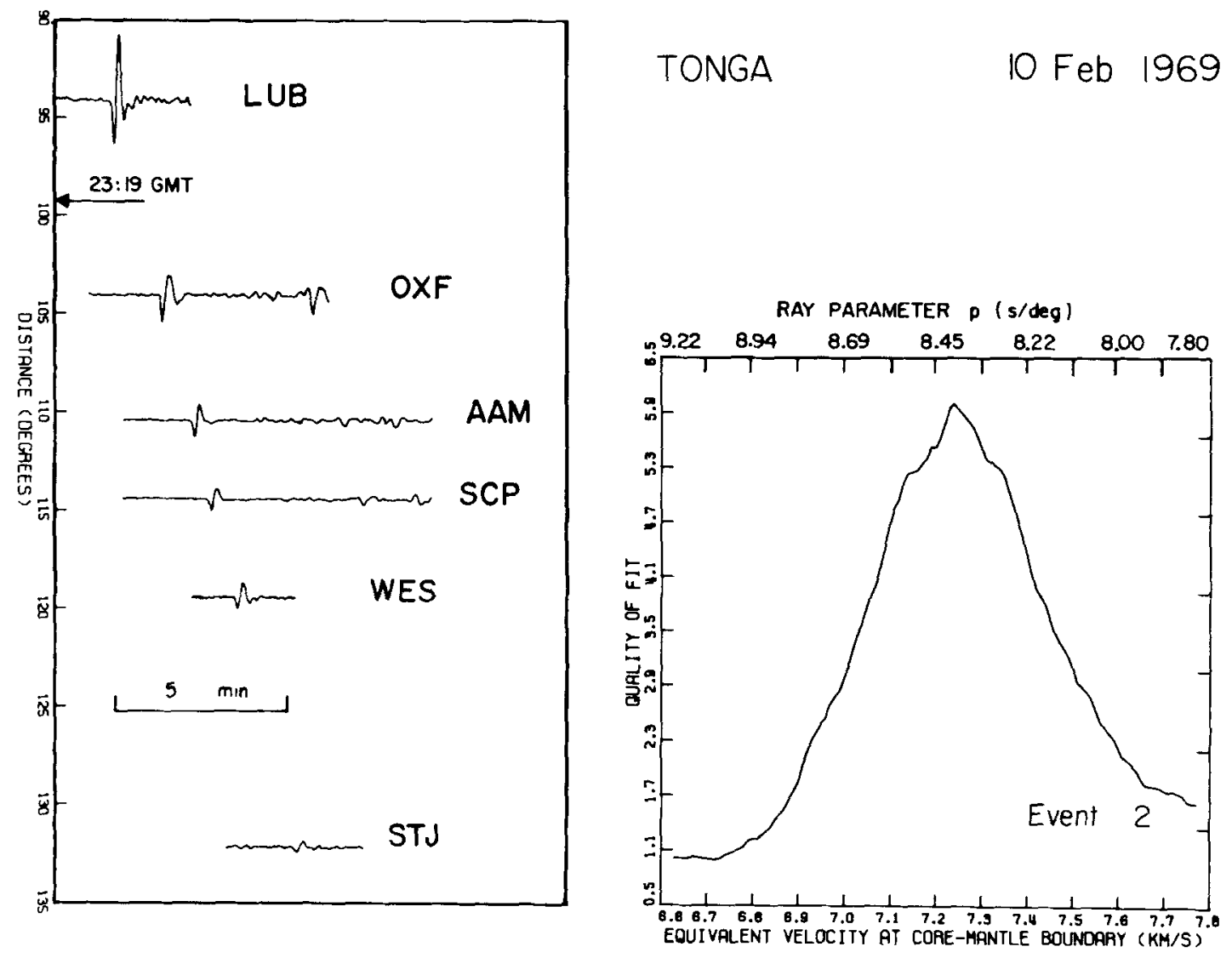

FIG. 4. Experimental profile for event 2 and variation of the cross-correlation, $F$, for event 2.

3 shows the (smoothed) variation of $F$ as a function of $p$. Its maximum is reached for $p_{0}=8.41 \mathrm{sec} / \mathrm{deg}$, a value in very good agreement with recent gross earth models (Gilbert and Dziewonski, 1975; Anderson and Hart, 1976). $F$ is 95 per cent of its maximum for $8.38<p<8.48 \mathrm{sec} / \mathrm{deg}$, and 90 per cent for $8.37<p<8.53 \mathrm{sec} / \mathrm{deg}$.

In principle, the theoretical shape of the diffracted pulse should change with distance (Chapman and Phinney, 1972), possibly leading to a bias in the cross correlation. However, the amount of distortion should be minimal for $\mathrm{SH}$ waves, as compared to $P$ and $S V$. Also, the excellent agreement in our study between the values of $p_{0}$ obtained at high frequency (by aligning the sharp onsets) and at low frequency shows that this effect is probably very small. 
Figures 4 and 5 show that similar values for $p_{0}$ are obtained for events 2 ( $p_{0}=8.39$ $\mathrm{sec} / \mathrm{deg})$ and $3\left(p_{0}=8.37 \mathrm{sec} / \mathrm{deg}\right)$. However, the relatively lower quality of the data does not permit a direct measurement of $d T / d \Delta$ from the onset of the wave, especially in the case of event 3 , whose source mechanism is very complex. Figure 6 shows an average of the three cross-correlation curves from Figures 3, 4, and 5. This suggests a value of $p_{0}=8.40 \mathrm{sec} / \mathrm{deg} . F$ is within 90 per cent of its maximum for $8.34<p<8.53 \mathrm{sec} / \mathrm{deg}$. We will write this as $p_{0}=8.43 \pm 0.1 \mathrm{sec} / \mathrm{deg}$. The error estimate of $0.1 \mathrm{sec} / \mathrm{deg}$ is somewhat arbitrary, since the 90 per cent level of the
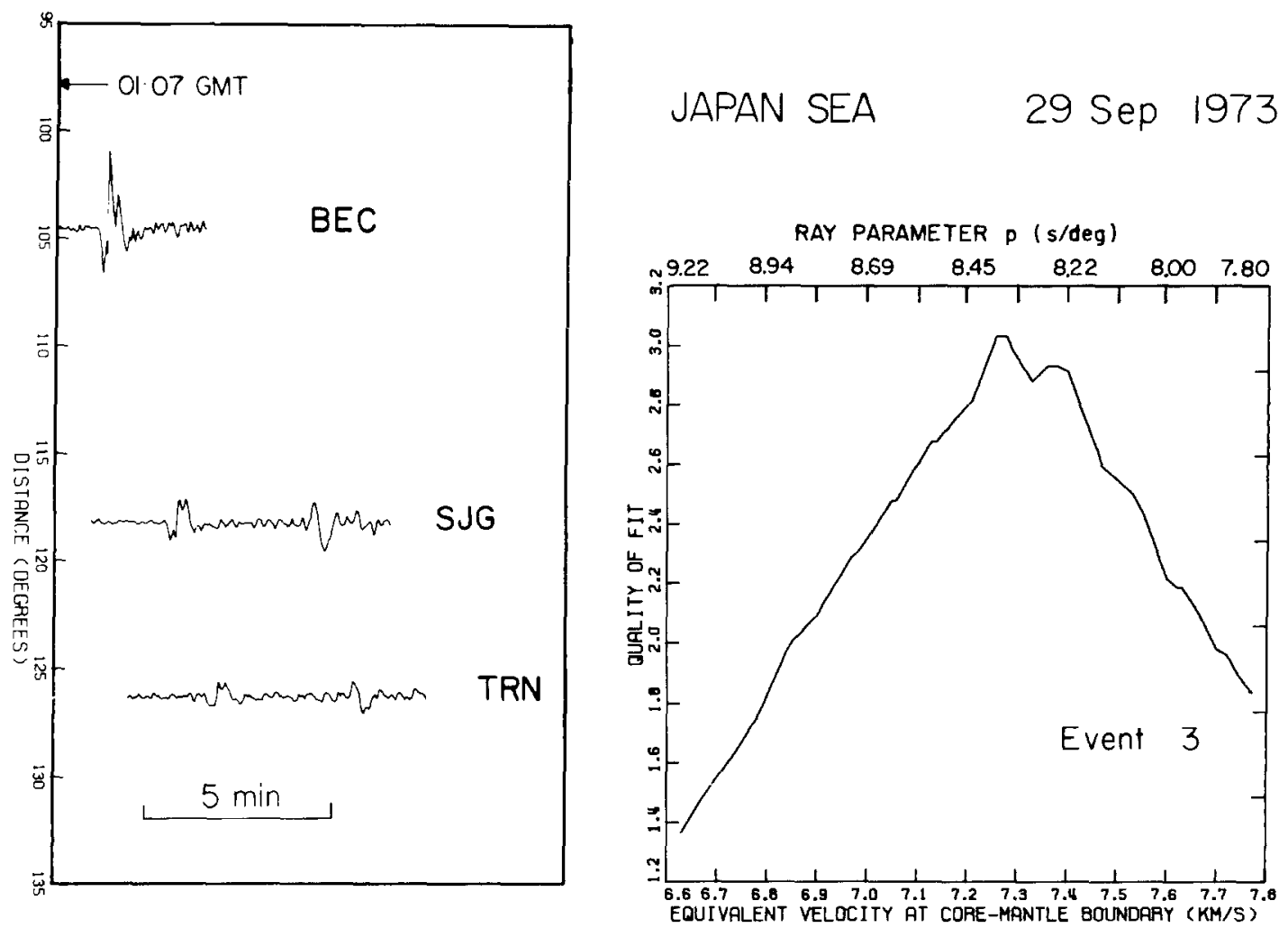

FIG. 5. Experimental profile and variation of the cross-correlation, $F$, for event 3 .

correlation function $F$ cannot be directly related to more conventional statistical error estimates.

\section{INTERPRETATION}

In the previous section, the slowness of $S d$ across three profiles of stations was found to be $d T / d \Delta=p_{0}=8.43 \mathrm{sec} / \mathrm{deg}$. Inferring the shear-wave velocity at the $\mathrm{CMB}, \beta_{c}$, from this slowness is not completely straightforward. If $S d$ is actually diffracted along the core-mantle interface, then $p_{0}=(\pi / 180) \cdot\left(r_{c} / \beta_{c}\right)$, where $r_{c}$ is the radius of the core $(3485 \mathrm{~km})$. However, some models proposed in the literature (Cleary et al., 1967; Mondt, 1977) have a low-velocity zone at the base of the mantle. This could lead to an $S H$ interface wave, propagated above the actual CMB. The information contained in $p_{0}$ would then be a tradeoff between the maximum $S$-wave 
velocity at the top of the hypothetical LVZ $\left(\beta_{\max }\right)$, and the thickness of the lowvelocity layer, $h$.

If a low-velocity zone is present above the CMB, then there should be a frequency dependent ray parameter for $S d$. At infinitely high frequencies, the ray parameter is directly related to $\beta_{\max }$

$$
\beta_{\max }=(\pi / 180) \cdot\left(r_{c}+h\right) / p_{0}
$$

If $p_{0}$ is interpreted in terms of an interface wave along the top of the low-velocity layer, above the CMB, one obtains $\beta_{\max }=7.44 \mathrm{~km} / \mathrm{sec}$ for a $100-\mathrm{km}$ thick lowvelocity zone, or $\beta_{\max }=7.64 \mathrm{~km} / \mathrm{sec}$ for $200 \mathrm{~km}$. [These thicknesses have been

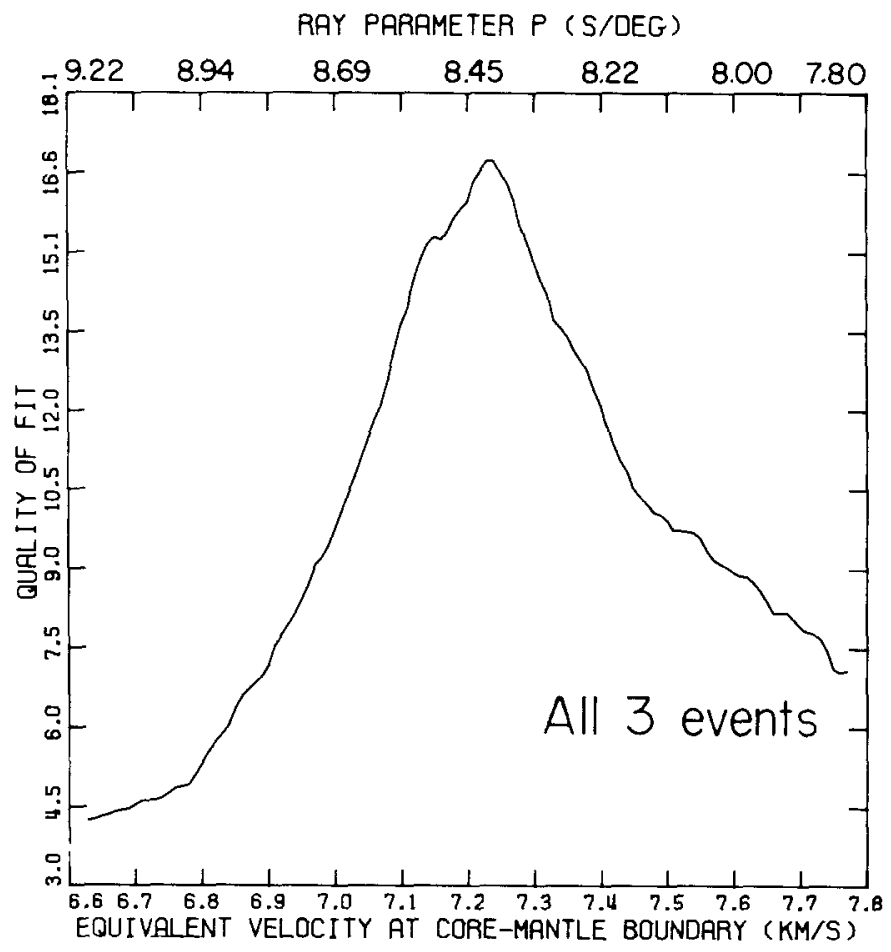

FIG. 6. Average of the three curves shown on Figures 3, 4, and 5. Each curve is weighed by the number of stations involved.

proposed by Mondt (1977).] These high values of $\beta_{\max }$ are difficult to reconcile with other seismological data such as $S$ travel times around $90^{\circ}$.

On the other hand, at finite frequencies, the wave slowness will average the $S$ wave velocity over a thickness on the order of one wavelength. the value of $p_{0}$ resudting from the cross correlation should then be frequency-dependent for a model with a low-velocity zone above the CMB. The excellent agreement between the high-frequency values of $p_{0}$ from the first arrivals $(8.44 \mathrm{sec} / \mathrm{deg})$ and the broadband values from cross correlation $(8.43 \pm 0.1 \mathrm{sec} / \mathrm{deg})$ suggests that there is no appreciable frequency dependence of $p_{0}$. To further investigate the possible frequency dependence, we ran the cross-correlation program after band-pass filtering the data from event 1 . An observable frequency dependence of $p_{0}$ should yield different peak 


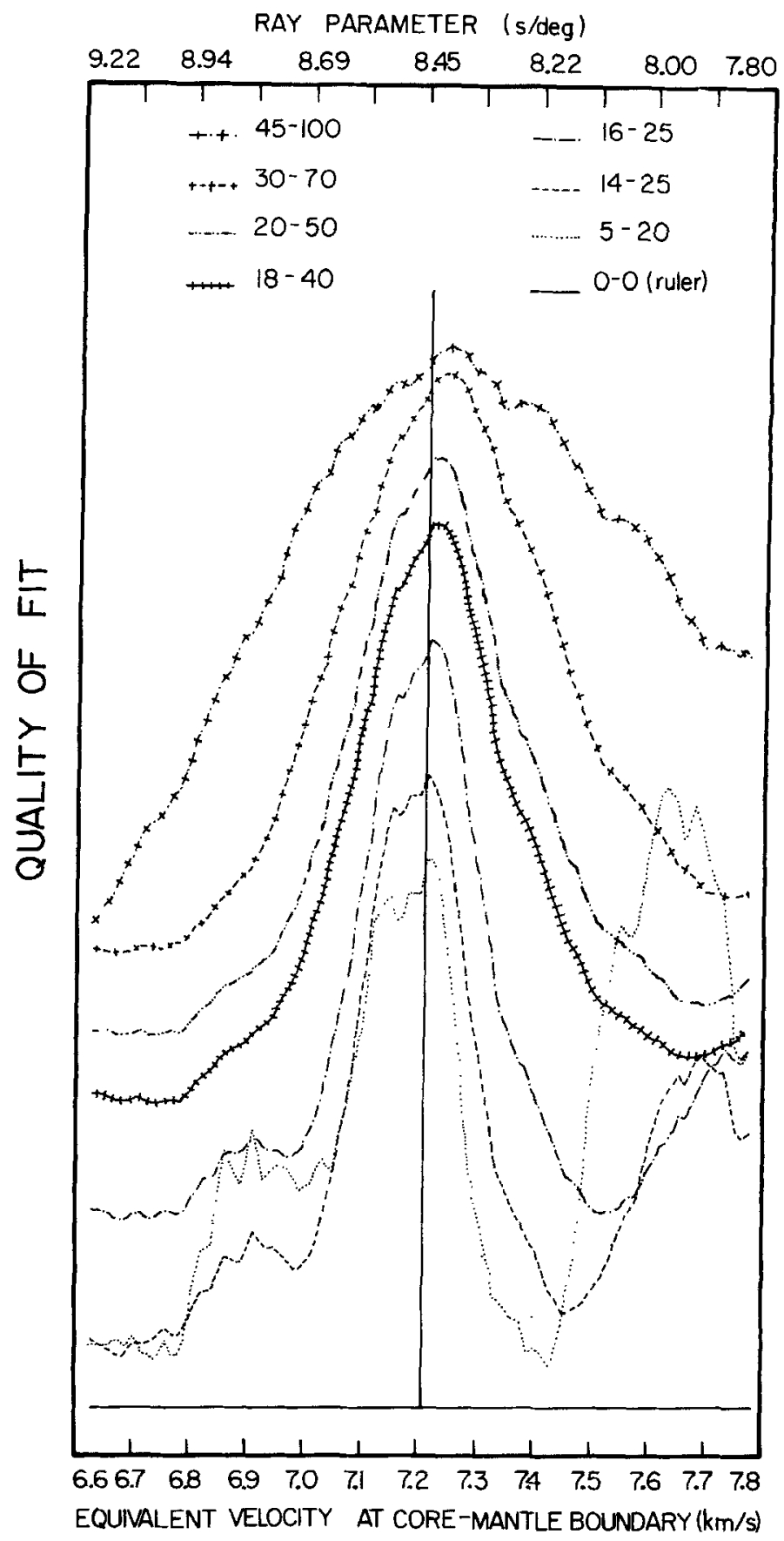

FIg. 7. Variation of the cross-correlation function, $F$, as a function of slowness (or, equivalently, velocity at the CMB) for event 1 , after band-pass filtering the records. The ranges of the band-pass filters (in seconds) are given in the key at the top of the figure. Superimposed (continuous line) is the value obtained by alignment of the onsets. The vertical scale is arbitrary, and the curves have been shifted upward to improve clarity. 
values at different frequencies. In fact, Figure 7 shows that $p_{0}$ is essentially frequency independent. Although a second peak appears around $p=7.95 \mathrm{sec} / \mathrm{deg}$ for the higher frequency band $(0.05$ to $0.2 \mathrm{~Hz})$, this is an artifact of the calculation. A periodicity of 0.4 to $0.5 \mathrm{sec} / \mathrm{deg}$ is expected in $F(p)$, because the average distance is $21^{\circ}$ and the predominant frequencies are 0.10 to $0.125 \mathrm{~Hz}$.

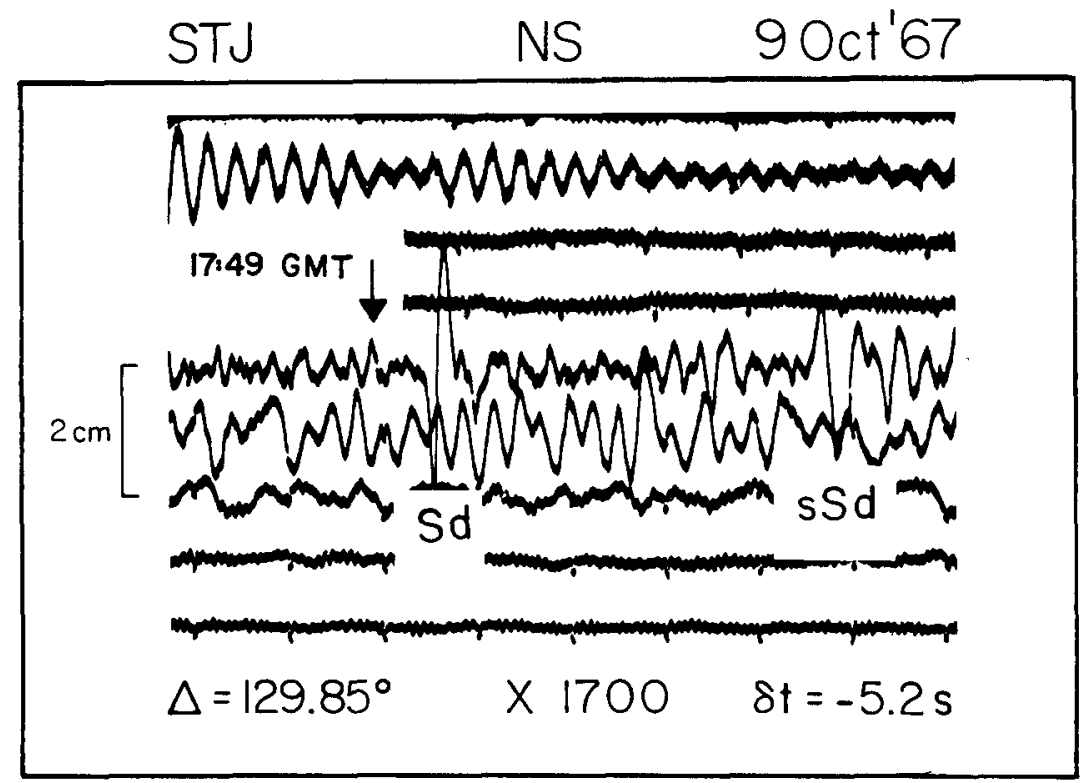

FIG. 8. A close-up of the phase $S d$ at STJ $\left(129.83^{\circ}\right)$ for event 1 . Note the sharp onset of the phase.

Another argument against the frequency dependence of $p_{0}$ comes from ray theory. For example, Dix (1961) and Helmberger (1968) have shown that the shape of a head wave is emergent in nature, the step-function response of a head wave being a ramp function. The profile of sharp onsets observed experimentally (Figure 8 is a close-up of the record at STJ, $\Delta=130^{\circ}$ ) is inconsistent with this model. We therefore consider that possible frequency dependence of $p_{0}$ can be ruled out. $\beta$ is considered

TABLE 3

Parameters of Models With a Rigid Layer at the TOP OF THE CORE

\begin{tabular}{cccc}
\hline Name & $\begin{array}{c}\text { Layer's Rigidity } \\
\left(\mathrm{dyn} / \mathrm{cm}^{2}\right)\end{array}$ & $\begin{array}{c}\text { Layer's Shear-wave } \\
\text { Velocity }(\mathrm{km} / \mathrm{sec})\end{array}$ & $\begin{array}{c}\text { Layer's Thickness } \\
(\mathrm{km})\end{array}$ \\
\hline$\beta \mathrm{C} 1$ & $4.0 \times 10^{11}$ & 2.00 & 50 \\
$\beta \mathrm{C} 2$ & $4.0 \times 10^{11}$ & 2.00 & 200 \\
$\beta \mathrm{C} 3$ & $1.0 \times 10^{11}$ & 1.00 & 35 \\
$\beta \mathrm{C} 4$ & $5.3 \times 10^{10}$ & 0.73 & 25 \\
\hline
\end{tabular}

as being essentially constant over a 100 - or $200-\mathrm{km}$ thick layer, and having a value of $7.22 \pm 0.1 \mathrm{~km} / \mathrm{sec}$.

\section{Synthetics of $S d$ : Preliminary Results}

We will investigate the velocity structure at the CMB by studying the decay of the $S d$ amplitudes along the profile of event 1 . At periods greater than 45 sec, to 
which our preliminary synthetics are restricted, it is not possible to resolve the $Q$ structure near the CMB, since the effect of $Q$ on $S d$ amplitudes is very small. Records filtered at $T \geqq 45 \mathrm{sec}$, therefore, provide a tool to investigate the purely elastic decay of $S d$ with distance, involving only propagation effects, and no attenuation. In this paper, we calculate synthetic seismograms by summing all the normal toroidal modes above $45 \mathrm{sec}$, using the procedure described by Kanamori and Cipar (1974). A zero-phase low-pass filter with linear amplitude response between $\frac{1}{45} \mathrm{~Hz}$ and $\frac{1}{70} \mathrm{~Hz}$ is used to compensate for the sharp cut-off at a 45 secperiod in the synthetics. A similar filter is also applied to the data. Results are shown in Figure 2, b (filtered data) and c (synthetics). The agreement is excellent.

Another important problem which can be investigated using synthetics is the possibility of nonzero rigidity of the core immediately below the CMB. This has

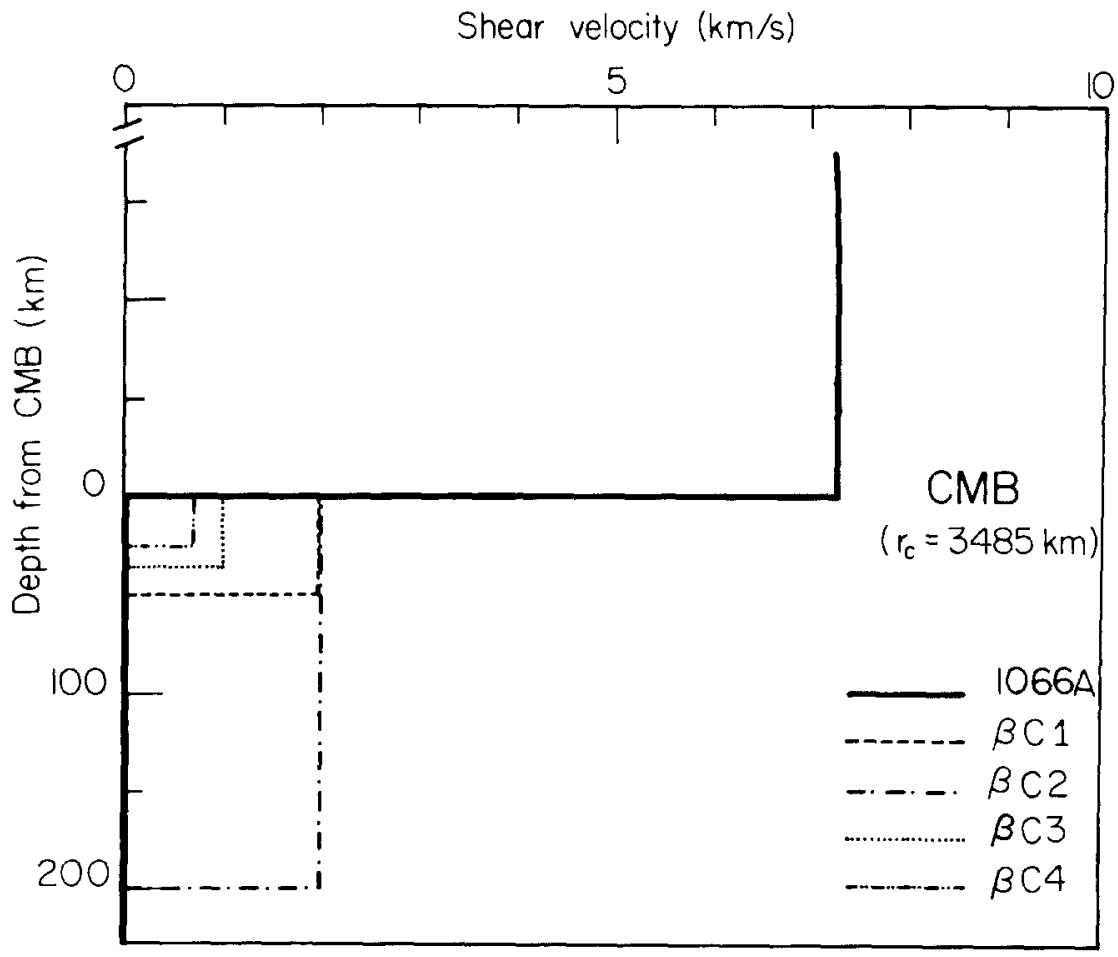

FIG. 9. Sketches of the models used to test the rigidity of the upper layers of the core. All of the models are identical to $1066 \mathrm{~A}$ in the mantle and below $r=3285 \mathrm{~km}$.

been proposed on the basis of the amplitude of $S$ and $S c S$ (Balakina and Vvedenskaya, 1962), of multiple $S c S$ (Sato and Espinosa, 1967) and of $P$ and $P c P$ (Ibrahim, 1971). We investigate whether the existence of a layer of slightly rigid material just below the CMB is compatible with $S d$ amplitudes.

For this purpose, we use four models, which are described in Table 3, and sketched in Figure 9. We do not use models with thinner rigid layers, since these must be investigated at higher frequencies. The values of the rigidity used in these models are in the range proposed by Balakina and Vvedenskaya (1962) and Ibrahim (1971). Synthetics were computed for event 1 , for which both excellent data and an accurate focal mechanism are available. Figure 10 compares the various synthetics with the data. In all four models, $S d$ disappears as a distinct seismic phase around $115^{\circ}$ : In models $\beta \mathrm{C} 1$ and $\beta \mathrm{C} 2$, the phase simply vanishes, while in models $\beta \mathrm{C} 3$ and $\beta \mathrm{C} 4$, 


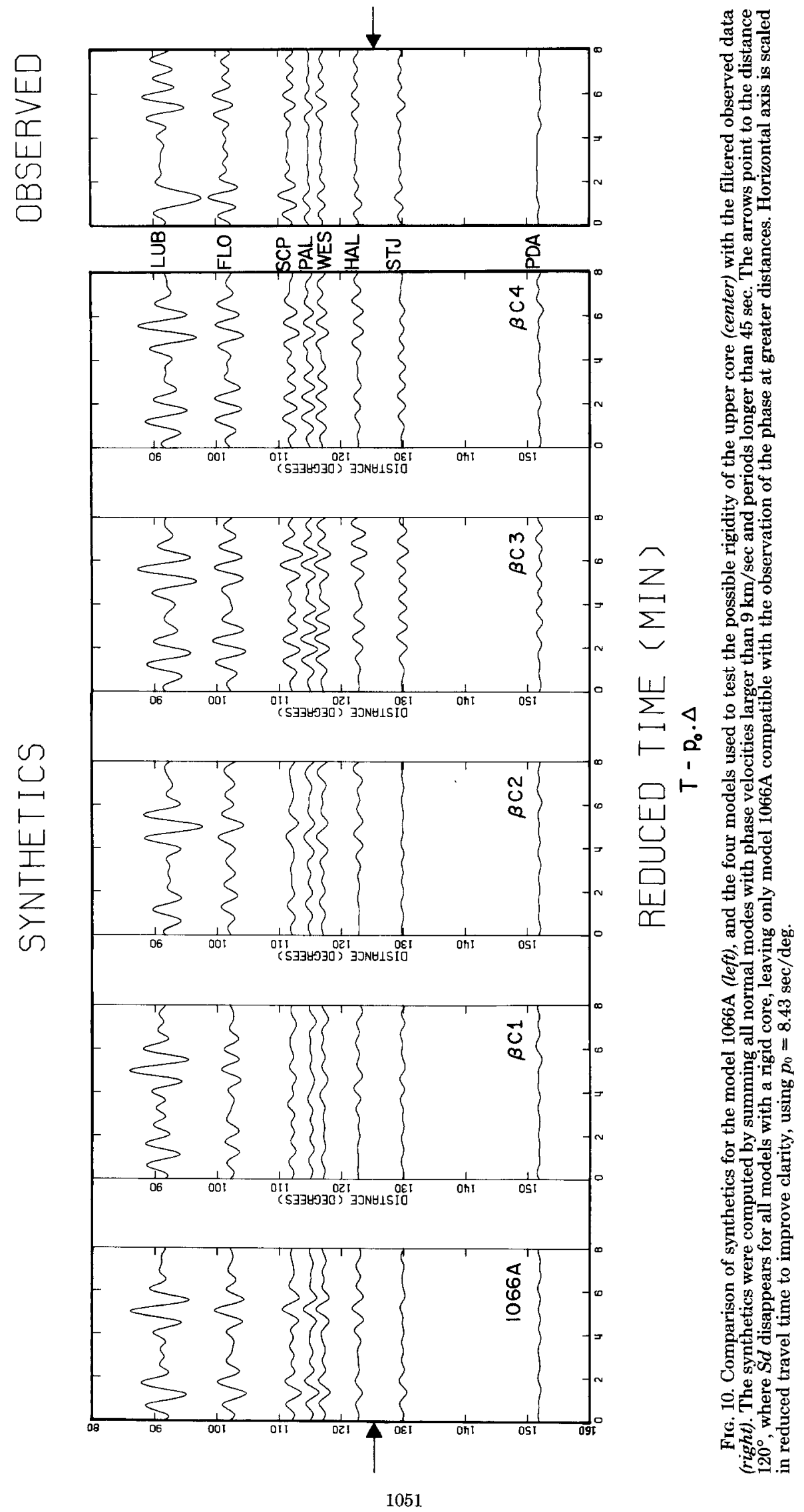


which have lower rigidities, the corresponding energy is smeared out, perhaps as a result of interference phenomena in the rigid layer. This faster amplitude decay is considered as being caused by seismic energy being trapped in the slightly rigid layer.

Among the models we have investigated, the only one compatible with the data in the frequency range $T \geqq 45 \mathrm{sec}$ is the conventional model 1066A. Thus, the present data suggests that the effect of CMB structure on waves with $T \geqq 45 \mathrm{sec}$ cannot be distinguished from those of a simple solid-liquid interface, with the maximum rigidity of the "liquid" below the CMB being smaller than $5 \times 10^{10}$ dynes/ $\mathrm{cm}^{2}$

Further investigation of the structure of the $\mathrm{CMB}$ will require the computation of synthetic seismograms at higher frequencies. If normal mode techniques are used, then the sum over discrete modes should be transformed into an integral over frequency (Kanamori and Stewart, 1976). The computation would then be very similar to the body wave synthetics for flat layered models which can be routinely calculated through modal superposition (Herrmann, 1977; Liao et al., 1978; Swanger and Boore, 1978). Reflectivity or generalized ray theory could also be used for $S d$ synthetics.

\section{CoNCLUSIONS}

By studying several profiles of $S H$ waves diffracted around the core $(S d)$, the average slowness is found to be $p_{0}=8.4 \pm 0.1 \mathrm{sec} / \mathrm{deg}$. If this value is interpreted as being due to the $S$ velocity just above the core-mantle boundary $\left(\beta_{c}\right)$, then it implies $\beta_{c}=7.22 \pm 0.1 \mathrm{~km} / \mathrm{sec}$, in contrast to previous models requiring a low- $S$ velocity zone just above the CMB.

Synthetic seismograms computed by summing normal modes may be used to study the effect of different CMB velocity structures on $S d$ wave shapes. We have used synthetics of $S d$ to show that certain models with nonzero $S$ velocity below the $\mathrm{CMB}$ are inadmissible. Also the frequency independence of the $S d$ ray parameter argues strongly against the possibility of a low-shear velocity zone immediately above the core mantle boundary.

\section{ACKNOWLEDGMENTS}

We thank Katsuyuki Abe, Don L. Anderson, John Cleary, Yoshio Fukao, Anton Hales, Don Helmberger, Hiroo Kanamori, and Seth Stein for discussion. The normal mode eigenfunctions for model 1066A were kindly made available by Freeman Gilbert and Ray Buland. This work was supported at Caltech by National Science Foundation Grant EAR 77-14675 and at Stanford by National Science Foundation Grant EAR78-03653.

\section{REFERENCES}

Anderson, D. L. and R. S. Hart (1976). An earth model based on free oscillations and body waves, $J$. Geophys. Res. 81, 1461-1475.

Anderson, D. L. and R. S. Hart (1978). Attenuation models of the earth, Phys. Earth Planet. Interiors 16, 289-306.

Balakina, L. M. and A. V. Vvedenskaya (1962). Variation of the elastic parameters and the density of matter at the boundary of the earth's core, Izv. Akad. Nauk SSSR, Ser. Geofiz. 11, 909.

Bolt, B. A., M. Niazi, and M. R. Sommerville (1970). Diffracted ScS and the shear velocity at the core boundary, Geophys. J. 19, 299-305.

Chapman, C. H. and R. A. Phinney (1972). Diffracted seismic signals and their numerical resolution, Methods Computational Phys. 12, 165-230.

Chung, W.-Y. and H. Kanamori (1976). Source process and tectonic implications of the Spanish deepfocus earthquake of March 29, 1954, Phys. Earth Planet. Interiors 13, 85-96.

Cleary, J. (1969). The $S$ velocity at the core-mantle boundary, from observations of diffracted $S, B u l l$. Seism. Soc. Am. 59, 1399-1405.

Cleary, J., K. Porra, and L. Read (1967). Diffracted S, Nature 216, 905-906. 
Dix, C. H. (1961). The seismic head pulse, reflection and pseudo-reflection pulses, J. Geophys. Res. 66, 2945-2951.

Furumoto, M. and Y. Fukao (1976). Seismic moment of great deep shocks, Phys. Earth Planet. Interiors 11, 352-357.

Gilbert, F. and A. M. Dziewonski (1975). An application of normal mode theory to the retrieval of structural parameters and source mechanisms from seismic spectra, Phil. Trans. Roy. Soc. London, Ser. A 278, 187-269.

Gutenberg, B. and C. F. Richter (1935). On seismic waves, II., Gerlands Beitr. Geophysik. 45, 280-360.

Hales, A. L. and J. L. Roberts (1970). The travel times of S and SKS, Bull. Seism. Soc. Am. 60, 461-489.

Helmberger, D. V. (1968). The crust-mantle transition in the Bering Sea, Bull. Seism. Soc. Am. 58, 179 214.

Herrmann, R. B. (1977). Research report of earthquake generated SH waves in the near-field and near regional field, Final Report, Contract DACW-39-0058, Waterways Experiment Station, Vicksburg, Miss.

Ibrahim, A.-B. K. (1971). The amplitude ratio $\mathrm{PcP} / \mathrm{P}$ and the core-mantle boundary, Pageoph 91, 114133.

Isacks, B. and P. Molnar (1971). Distribution of stresses in the descending lithosphere from a global survey of focal mechanism solutions of mantle earthquakes, Rev. Geophys. Space Phys. 9, 103-174.

Jacobs, J. A. (1975). The Earth's Core, Academic Press, London, 263 pp.

Kanamori, H. and J. J. Cipar (1974). Focal process of the great Chilean earthquake, May 22, 1960, Phys. Earth Planet. Interiors 9, 128-136.

Kanamori, H. and G. S. Stewart (1976). Mode of the strain release along the Gibbs Fracture Zone, MidAtlantic Ridge, Phys. Earth Planet. Interiors 11, 312-332.

Kind, R. and G. Muller (1977). The structure of the outer core from SKS amplitudes and travel times, Bull. Seism. Soc. Am. 67, 1541-1554.

Lehmann, I. (1953). On the shadow of the earth's core, Bull. Seism. Soc. Am. 43, 291-306.

Liao, A. H., F. Schwab, and E. Mantovani (1978). Computation of complete theoretical seismograms for torsional waves, Bull. Seism. Soc. Am. 68, 317-324.

Mikumo, T. and T. Kurita (1968). Q distribution for long-period P waves in the mantle, J. Phys. Earth 16, 11-29.

Mitchell, B. J. and D. V, Helmberger (1973). Shear velocities at the base of the mantle from observations of $\mathrm{S}$ and $\mathrm{ScS}, \mathrm{J}$. Geophys. Res. 78, 6009-6020.

Mondt, J. C. (1977). SH waves: Theory and observations for epicentral distances greater than 90 degrees, Phys. Earth Planet. Interiors 15, 46-59.

Niazi, M. (1969). Source dynamics of the Dasht-e-Bayāz earthquake of August 31, 1968, Bull. Seism. Soc. Am. 59, 1843-1861.

Okal, E. A. and D. L. Anderson (1975). A study of lateral inhomogeneities in the upper mantle by multiple $\mathrm{ScS}$ travel time residuals, Geophys. Res. Letters 2, 313-316.

Richards, P. G. (1970). A contribution to the theory of high frequency elastic waves, with applications to the shadow boundary of the earth's core, Ph.D. Thesis, California Institute of Technology, Pasadena.

Sato, R. and A. F. Espinosa (1967). Dissipation in the earth's mantle and rigidity and viscosity in the earth's core determined from waves multiply reflected from the mantle-core boundary, Bull. Seism. Soc. Am. 57, 829-856.

Scholte, J. G. J. (1956). On seismic waves in a spherical earth, K. Ned. Meteorol. Inst. Meded. Ver. 65, $1-55$.

Sipkin, S. A. and T. H. Jordan (1976). Lateral heterogeneity of the upper mantle determined from the travel times of multiple ScS, J. Geophys. Res. 81, 6307-6320.

Swanger, H. J. and D. M. Boore (1978). Simulation of strong motion displacements using surface-wave modal superposition, Bull. Seism. Soc. Am. 68, 907-922.

Yoshida, M. and M. Tsujiura (1975). Spectrum and attenuation of multiply reflected core phases, $J$. Phys. Earth (Tokyo) 23, 31-42.

SEISMOLOGICAL LABORATORY

California Institute of Technology

Pasadena, California 91125 (E.A.O., R.J.G.)

Contribution No. 2985
DEPARTMENT OF GEOPHYSICS

STANFORD UNIVERSITY

Stanford, California 94305 (R.J.G.) 